\title{
Wide-Area Impact Damage Evaluation with Sonic Infrared Imaging NDE in Advanced Composite Structures
}

\author{
by X. Han*, Justin M. Ar-Rasheed*, D. Zhang*, A. Lubowicki ${ }^{*}$, L. Favro**, and G. Newaz ${ }^{* * *}$ \\ Department of Electrical and Computer Engineering, Wayne State University, Detroit, MI 48202, \\ USA, xhan@eng.wayne.edu \\ ** Department of Physics and Astronomy, Wayne State University, Detroit, MI 48202, USA \\ *** Department of Mechanical Engineering, Wayne State University, Detroit, MI 48202, USA
}

\begin{abstract}
Sonic Infrared (IR) imaging is a relatively new member in the NDE technology family. This method combines a short pulse of ultrasound excitation, typically a fraction of a second, and infrared imaging. It has been shown its great advantage as a NDE technology to detect defects such as cracks in metal/metal alloy structures. As an imaging technology, Sonic IR is capable of wide area evaluation. Composite materials have been used in broader applications due to its light weight and stiffness. However, composites suffer more to impact than metal structures. Such damage in composites may not present at the surface but severe damage could have occurred in deeper layers. In such case, the structure strength is greatly weakened. We have been studying impact damages in composite structures with Sonic Infrared Imaging, and evaluating how structures affect thermal signatures in defect characterization. In this paper, we will present our study on composite panels containing structures such as doublers and beams or stiffeners, which were subjected to impacts from varying energy projectiles.
\end{abstract}

Keywords: Sonic IR imaging, NDE, impact damage, carbon-fiber enforced composite structures

\section{Introduction}

Composite materials offer a wide choice of matrix and reinforcements for various applications that span from marine to automotive and aerospace applications. The wide choice of reinforcements and matrices also offer cost advantages for specific applications where semi-structural and structural parts can be manufactured to meet performance requirements. For those reasons, composites have provide themselves broad applications in various industries. However, materials such as carbon fiber composites (CFC) has strength weakness, in which if damage, such as delamination, occurs, it could be deadly in critical structures like in aircraft if it's not identified in time. Sonic IR Imaging has shown its capability as a fast, wide area NDE method. We have been studying impact damages in composite structures and evaluating how structures affect thermal signatures in defect characterization. In this paper, we will present our results on composite samples which contains different structures. The samples were subjected to impacts from varying energy projectiles and resulted in different levels of damages.

\section{Some Results and Brief Discussion}

Composite aircrafts have many different substructures under the fuselage's outer skin. These stringers, doublers, etc., can vary considerably in their construction and may affect sound propagation differently. Of special interest is the distance that sound travels in composite structures. This affects the maximum range from the sound injection point to the furthest detectible defect. One of the samples we have used for studying is a composite panel of $152 \mathrm{~cm}$ long and $54 \mathrm{~cm}$ wide. This panel has doublers, hat-beams over doublers, and regular skin thickness. It also has distributed impacted points with a distribution of energies in the impacts. These impact energies were chosen so that they would probably cause internal damage, but would cause, at most, barely visible surface damage. In fact, almost none of the impacts could be detected visually, even in glancing light. This was in spite of the fact that the samples were painted with glossy white aircraft paint that will show the slightest surface dimple in such light. Photos of the panel's front and back sides are shown in Figure 1.

Figure $2 \mathrm{a}$ is a Sonic IR image of the panel showing the impact damage detected with the $20 \mathrm{kHz} 0.8$ second ultrasound sound pulse injected at the right side edge of the panel. The image shows several impact damages underneath the top layers. In addition, temperature time plots over six selected impact damage spots are shown in Figure $2 \mathrm{~b}$. These data can be used to determine how much the signal from any damage rose above the background and can be used for quantitative evaluation of impact damages. 

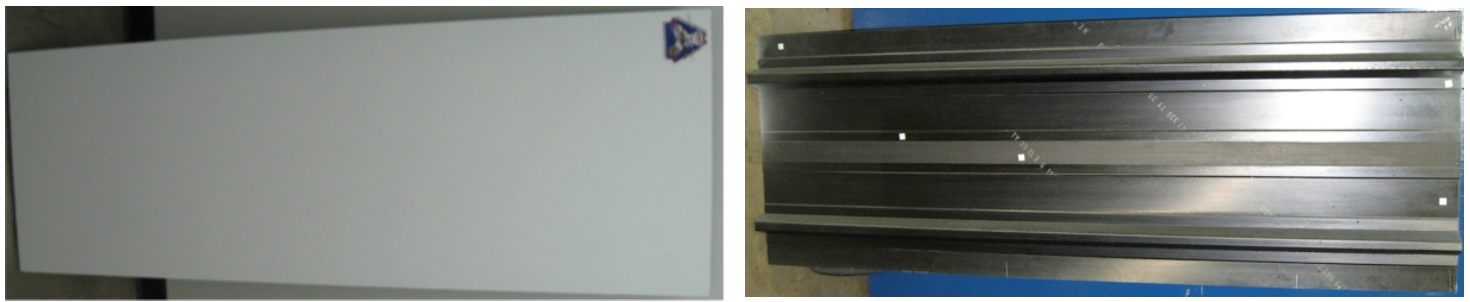

Figure 1. Photos of a composite panel's front and back side. The panel is $152 \mathrm{~cm}$ long and $54 \mathrm{~cm}$ wide.

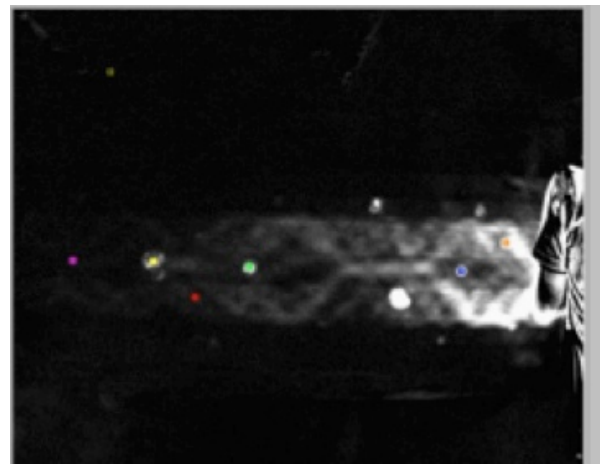

a

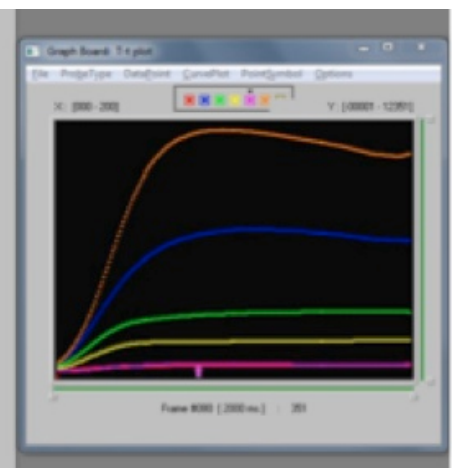

b

Figure B2. IR image showing the impact damage and temperature time plots over six selected spots.

It is clearly shown that Sonic IR can image a wide area within very short time to identify damage in composites. However, sound waves do have much larger damping in composite materials than in metal and metal alloys. Detailed discussion and data will be presented in the paper.

\section{ACKNOWLEDGMENTS}

This work was sponsored by the Federal Aviation Administration William J. Hughes Technical Center under Contract Number DTFACT-08-C-00043 with Agency Contract Number 437-65061, and in part by Wayne State University. The impact samples were provided by Robert Barry from Bell Helicopter.

\section{REFERENCES}

[1] L.D. Favro, Xiaoyan Han, Zhong Ouyang, Gang Sun, Hua Sui, and R.L. Thomas, "Infrared imaging of defects heated by a sonic pulse", Review of Scientific Instruments, Vol. 71, P2418-2421, June 2000.

[2] Xiaoyan Han, Wei Li, and Zhi Zeng, L.D. Favro, R.L. Thomas, "Acoustic Chaos and Sonic Infrared Imaging", Applied Physics Letters, vol.81, 17, pp3188-3190, October 21, 2002

[3] Xiaoyan Han, Zhi Zeng, Wei Li, Mahmad Islam, Jianping Lu, Vera Loggins, E. Yitamben, L.D. Favro, G. Newaz, and R.L. Thomas, "Acoustic Chaos for Enhanced Detectability of Cracks by Sonic Infrared Imaging", Journal of Applied Physics, Vol.95, No.7, pp3792-3797, April 1, 2004

[4] Qi He, Xiaoyan Han, "Crack Detection Using Sonic Infrared Imaging in Steel Structures: Experiments and Theory of Heating Patterns", SPIE, Smart Structures and Materials, Vol. 7292, 72920Q-1-7, 2009

[5] Xiaoyan Han, Qi He, and Ding Zhang, Mike Ashbaugh, Lawrence D. Favro, Golam Newaz, Robert L. Thomas, "Damage Threshold Study Of Sonic IR Imaging On Carbon-Fiber Reinforced Laminated Composite Materials", Review of Progress in Quantitative Nondestructive Evaluation, p525 -531, Vol. 32, 2013 